\title{
JOURNÉES ÉQUATIONS AUX DÉRIVÉES PARTIELLES
}

\section{RICHARD BEALS}

Propagation de singularités pour les opérateurs du type $D_{t}^{2}-\square_{b}$

Journées Équations aux dérivées partielles (1980), p. 1-3

$<$ http://www.numdam.org/item?id=JEDP_1980 A19 0>

(C) Journées Équations aux dérivées partielles, 1980, tous droits réservés.

L'accès aux archives de la revue «Journées Équations aux dérivées partielles » (http://www. math.sciences.univ-nantes.fr/edpa/) implique l'accord avec les conditions générales d'utilisation (http://www.numdam.org/conditions). Toute utilisation commerciale ou impression systématique est constitutive d'une infraction pénale. Toute copie ou impression de ce fichier doit contenir la présente mention de copyright.

\section{NumDam}

Article numérisé dans le cadre du programme Numérisation de documents anciens mathématiques http://www.numdam.org/ 


\section{PROPAGATION DE SINGULARITES POUR LES}

OPERATEURS DU TYPE $D_{t}^{2}-\mathrm{D}_{\mathrm{b}}$

par R. BEALS

Sur une variété compacte $M$ on considère un opérateur (pseudo-) différentiel $P$ du second ordre, de symbole principal p. On garde toujours les hypothèses suivantes : (H 1) $\quad \int_{M} \bar{u} P u d \mu \geqslant 0$ pour chaque $u \in C^{\infty}(M)$, où $\mu$ est une densité positive lisse. $\Sigma=\{\mathrm{p}=\mathrm{O}\} \subset \mathrm{X}=\mathrm{T}^{*} \mathrm{M} \backslash \mathrm{O}$ est une sous-variété symplectique sur laquelle p s'annule exactement à l'ordre 2 .

(H 3) P est hypoelliptique avec perte d'une dérivée (si $\sum \neq \varnothing$ ).

Notons que d'après Boutet de Monvel [1], on sait qu'avec (H 2), la condition (H 3) équivaut à une condition explicite sur le symbole sous-principal de $\mathrm{P}$ sur $\sum$. L'exemple le plus important est (la partie de) l'opérateur $\square_{b}$ au bord d'un domaine complexe strictement pseudo-convexe.

D'après (H 1), p est positif ou nul. Alors $q=\sqrt{p}$ appartient à $C^{\infty}(X \backslash \Sigma)$ et $q$ engendre un flot hamiltonien $\Phi_{s}=\exp \left(s_{H}\right) \operatorname{sur} x \backslash \Sigma$.

Proposition : Le flot $\left(\Phi_{S}\right)_{S} \in \mathbb{R}$ se prolonge en un groupe d'homéomorphismes de $x$ (notés toujours $\Phi_{S}$ ) qui fixent les points de $\Sigma$.

$$
\text { On considère aussi les groupes d'homéomorphismes de } \mathrm{T}^{*} \mathbb{R} \times \mathrm{x}=\mathbb{R} \times \mathbb{R} \times \mathrm{X}
$$
définis par

$$
\Psi_{s}^{ \pm}(t, \tau, w)=(t+s, \tau, \Phi \pm s(w)), \quad w \in X
$$

Posons $Q=P^{1 / 2}$. La factorisation $L=D_{t}^{2}-P=L^{+} L^{-}=L^{-} L^{+}$, où $L^{ \pm}=D_{t} \pm Q$, réduit l'étude de l'équation des ondes pour P à l'étude de l'équation de Schrödinger pour $Q$. Q engendre un groupe unitaire $U(s)=\exp (i s Q)$, et on peut montrer que les opérateurs s'étendent à $\mathbf{8} \cdot(\mathbf{M})$. 
Théorème 1 : Soit $f \in \mathcal{\prime}^{\prime} \mathrm{A}^{\prime}(M)$. Alors les spectres singuliers (fronts d'onde) satisfont à $W F(U(s) f)=\Phi_{-s}(W F(f))$.

$$
\text { Définissons car } L^{ \pm}=\left\{(t, \tau, w) \in T^{*} \mathbb{R} \times x: \tau \pm q(w)=0\right. \text {. }
$$

$\underline{\text { Théorème } 2}$ : Soit $f \in \mathscr{D}^{\prime}(\mathbb{R} \times M)$. Posons $\Gamma=W F(f) \backslash W F(L f), \Gamma^{ \pm}=\Gamma \backslash$ car $L^{\mp}$, $\Gamma_{0}=\Gamma \cap \operatorname{car} L^{+} \cap \operatorname{car} L^{-}$. Alors $\Gamma$ est la réunion disjointe $\Gamma=\Gamma^{+} U \Gamma^{-} U \Gamma_{0}$. De plus, $\Gamma^{+}$et $\Gamma_{0}$ (resp. $\Gamma^{-}$et $\Gamma_{0}$ ) sont contenus dans car $\mathrm{L}^{+}$(resp. $\mathrm{car}^{-}$) et ils sont invariants par le flot $\left(\Psi_{s}^{+}\right)$(resp. $\left(\Psi_{s}^{-}\right)$).

Signalons que dans le cas où $\operatorname{codim} \Sigma=2$, le Théorème 2 est dû à $B$. Lascar [2] et à Melrose [3] - Dans le cas classique $\sum=\varnothing$, l'opérateur P est elliptique, la racine carrée $Q$ est pseudodifférentiel et classique, et les Théorèmes 1 et 2 se déduisent facilement du fait que pour n'importe quel opérateur pseudodifférentiel classique $\mathrm{B}$, les opérateurs $\mathrm{B}(\mathrm{s})=\mathrm{U}(\mathrm{s}) \mathrm{BU}(-\mathrm{s})$ restent pseudodifférentiels et leurs symboles principaux satisfont à $b(s)=b \circ \Phi_{s}$. De plus, ce fait peut être démontré facilement par récurrence à partie de l'identité :

$$
\begin{aligned}
B(s) & =B_{s}+\int_{0}^{S} \frac{d}{d r}\left[U(r) B_{s-r} U(-r)\right] d s \\
& =B_{s}+\int_{0}^{S} U(r) C_{s-r} U(-r) d s
\end{aligned}
$$

où les $B_{S}$ ont pour symboles (complets)b ${ }^{\circ} \Phi_{s^{\prime}}$ puisque les $C_{r}$ sont des opérateurs pseudodifférentiels d'ordre:ordre (B) - 1 .

Dans le cas général $Q$ est un opérateur pseudodifférentiel non-classique, mais classique en dehors de chaque voisinage conique de $\sum$. Alors $B$ (s) est encore pseudodifférentiel si son symbole complet ne rencontre pas $\sum$. Pour aller jusqu'à $\Sigma$ on définit le support essentiel de $B: \mathbf{D}^{\prime}(\mathrm{M}) \rightarrow \boldsymbol{D}^{\prime}(\mathrm{M})$ comme le plus petit fermé conique $\Gamma \subset x$ tel que WF(f) $\cap \Gamma=\varnothing$ implique $B f \in C^{\infty}(M)$. Alors tout marche comme auparavant dès qu'on a le lemme suivant, qui est aussi démontré par la récurrence ci-dessus.

$\underline{\text { Lemme }}$ : Soit $w \in \sum$ et soit $V$ un voisinage conique de $w$. Alors pour chaque $s_{0}>0$ il existe un opérateur pseudodifférentiel B, elliptique en $w$, tel que le support essentiel de $\mathrm{B}(\mathrm{s})=\mathrm{U}(\mathrm{s}) \mathrm{BU}(-\mathrm{s})$ soit contenu dans $\mathrm{V}$ pour $|\mathrm{s}| \leqslant \mathrm{s}_{0}$. 


\section{Références}

[1] L. Boutet de Monvel : Comm. Pure Appl. Math. 27 (1974).

[2] B. Lascar, à paraître. (Exposé à Orsay 10/1979).

[3] R. B. Melrose, exposé au séminaire Goulaouic-Schwartz 1980. 